\title{
MULTIPLE RHYTHMS AND SWITCHES IN THE NERVOUS SYSTEM
}

\author{
N. KOPELL \\ Department of Mathematics and Statistics, Boston University, Boston MA 02215 \\ D. PERVOUCHINE \\ Center for BioDynamics, Boston University, Boston MA 02215 \\ H. G. ROTSTEIN \\ Department of Mathematics and Statistics, Boston University, Boston MA 02215 \\ T. NETOFF \\ Department of Biomedical Engineering, Boston University, Boston MA 02215
}

\section{WHITTINGTON}

School of neurology, Neurobiology and psychiatry, University of Newcastle, Newcastle upon Tyne NE2 $4 H H, U K$

\section{T. GLOVELI}

Institute of Neurophysiology, Charite-University Medicine, Berlin 10117, Germany

\begin{abstract}
Networks of neurons in the nervous system can produce a variety of temporal patterns of different frequency; the same network can produce different rhythms at different times, or several rhythms at one time. We focus here on the gamma $(30-90 \mathrm{~Hz})$ and theta (4-12 Hz) rhythms produced in the hippocampus, a part of the nervous system critical for learning and recall. We discuss experiments and models that suggest that there are separate subnetworks that produce the different rhythms; the sharing of components of the networks induces competition between the rhythms, which can lead to suppression of one of the rhythms, or nesting of the rhythms. We show how low-dimensional maps can help to understand the properties of the cells and networks to allow this to happen.

Keywords: neural dynamics, gamma rhythm, theta rhythm, hippocampus, lowdimensional maps
\end{abstract}

\section{Rhythmic dynamics in the nervous system}

The nervous system produces dynamics at all states of wake and sleep. These can be detected via EEG and MEG measurements in non-invasive ways, and through invasive electrophysiology techniques. All these techniques indirectly record the activity of electrical currents that are created by neurons in the brain, signaling to other 
neurons. These electrical and magnetic signals can be analyzed for their spectral content, and it has been found that the spectral content varies with the cognitive state of the subject: certain frequency ranges, such as the gamma frequency (roughly $30-90 \mathrm{~Hz}$ ) are associated with attention, active processing of early sensory signals, short term memory and other behavioral situations. The theta rhythm (roughly $4-12 \mathrm{~Hz}$ in rats and $4-8 \mathrm{~Hz}$ in humans) is seen in active exploration in rats, and is believed by many to be important for the recording and feedback of memory, especially memory of sequences of places or events.

The mathematical questions associated with these rhythms concern their biophysical origins, and how the same bits of tissue can, in different behavioral situations, produce different rhythms or combination of rhythms. By piecing together the biophysical substrate of the rhythms, one is then in a position to start investigating how the networks can process different kinds of spatially and temporally patterned signals; such information is critical to understanding how the brain makes use of these dynamics for cognitive function.

Many of the rhythms that are seen in behaving animals have been replicated in vitro (in slices of brain tissue). This allows the use of much more powerful measures to discover the biophysical nature of the different rhythms. Much of the work that has been done deals with single spectral bands. However, recently, some labs have been able to reproduce a combination of rhythms that appear very much as they do in vivo. This gives us a window into how the nervous system may be using interactions of rhythms to process information. One of the most widespread pair of interacting rhythms is the gamma/theta pair (Chrobak et al. , 2000), and that is the focus of this talk.

\section{Gamma, Theta and in vitro preparations}

In vitro preparations are different from one part of the nervous system to another, and the anatomical focus of this talk is on a part of the hippocampus known as CA3. This is a section of the so-called "hippocampal loop", in which signals come from the neocortex, go around this loop (with some further possible inputs and outputs), and then go back to the neocortex, presumably in a different form. This loop is believed to be crucial in the encoding of new memories. The CA3 region is known in vivo to produce both theta and gamma in a nested manner. In order to address what the system might be doing with those rhythms, it is helpful to understand what creates them, and how they related to one another. A recent breakthrough in this came from the work of Gloveli et al. (2005) who showed that a network in a slice that produces both can in some sense be parsed into interacting and overlapping gamma and theta networks. The idea, though not the work, was simple: By slicing in the standard transverse direction, one gets a gamma rhythm, in the longitudinal direction a theta rhythm, and in a direction in between, a nested rhythm. The modeling and mathematical issues addressed in this paper concern how this comes about. The essential idea is that the full network is composed of different 
kinds of cells, and different subnetworks of those cells are responsible for different rhythms. Under different conditions - either the angle of the slice or modulatory changes in the chemical soup - different subnetworks can take over and produce different rhythms. We believe this is a general principle in the nervous system. We first describe mechanisms separately for the gamma and theta rhythm, then return to this example to talk about how they interact in a larger network.

\section{The Mathware: Voltage-gated conductance equations}

The simulations we discuss come mainly from the so-called Hodgkin-Huxley equations or, more generally, "voltage-gated conductance equations" (Dayan \& Abbott, 2005). These are equations for a single neuron, or a network of neurons. For each cell, the main equation is for the voltage difference across the cell membrane, and the equation represents conservation of currents passing through the membrane, balanced by a capacitance current. Each of the currents is generated by single kind of ion (or combination of ions) that pass through molecular "channels" that open and close with voltage-dependent kinetics. For each cell, the current conservation equations are supplemented by other equations that describe the opening and closing of channels as the voltage changes. The full equations are PDEs, describing the voltage across the spatially extended cell. However, in simplified models, the spatial extension is often ignored, giving rise to a set of equations known as a "point" neuron. In this paper, most simulations use point neurons. The main equation is:

$$
C \frac{\partial v}{\partial t}=-I_{i o n}+\nabla^{2} v-\sum I_{\text {synapse }}
$$

where

$$
I_{\text {ion }}=g m^{j} h \cdot\left(v-V_{R}\right) .
$$

Here each ionic current is given by Ohm's law: it is a product of the conductance $g m^{j} h$, which is the inverse of the resistance, and a driving force $v-V_{R}$. The latter acts like a battery with a "reversal potential" that depends on the kind of ion; $g$ is a constant, and $m$ and $h$ are gating variables, describing channels in the membrane that open an close in a voltage dependent manner, according to differential equations of a form

$$
\frac{d x}{d t}=\left(x_{\infty}(v)-x\right) / \tau(v)
$$

The functions $x_{\infty}(v)$ are sigmoid as in figure 1.

Each neuron may have a different set of conductances and different types of neurons generally do have such differences. Equations for a network of neurons consists of separate sets of equations for each cell, coupled via the voltage equation with other currents labeled $I_{\text {synapse }}$. The coupling currents can be excitatory or inhibitory; roughly speaking, excitatory currents increase the voltage, making it easier for the receiving (post-synaptic) cell to fire, while inhibitory currents make it harder for the post-synaptic cell to fire. 

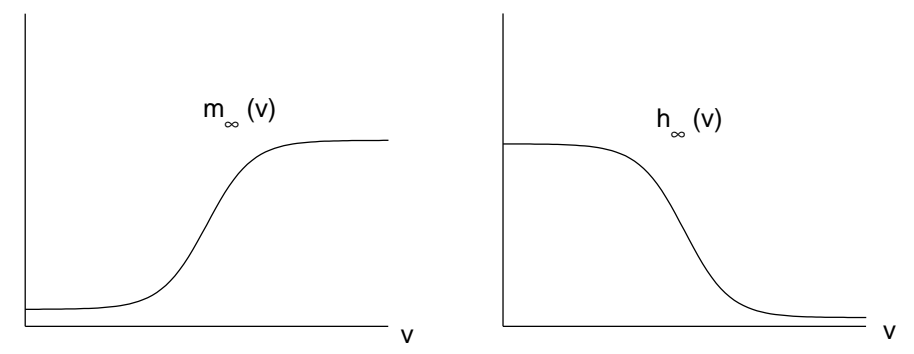

Fig. 1. Activation curves for gating variables. The vertical axis is the fraction of channels open when the voltage is held at a given value. Such a curve is usually monotonic, and can either increase or decrease with voltage.

\section{Some mechanisms for the gamma rhythm}

There are (at least) several different biophysical mechanisms that produce a gamma rhythm. Two are discussed here. Only one is directly important for the CA3 story, but the other provides a contrast to another phenomenon that is central to the story.

Unlike some other rhythms, gamma is very much an inhibition- based rhythm (Whittington et al. , 2000). The reason comes basically from how common inhibition acts to synchronize target cells. It is illustrated by the simplest form of neural equations, the "integrate-and-fire" neuron, with an additional term for inhibition.

$$
\begin{aligned}
& \frac{\partial v_{1}}{\partial t}=I-v_{1}-g_{s y n} e^{-t / \tau} \\
& \frac{\partial v_{2}}{\partial t}=I-v_{2}-g_{s y n} e^{-t / \tau}
\end{aligned}
$$

The inhibition is modeled here by a synapse that turns on instantaneously, and then decays exponentially with time constant $\tau$. The essential point is that the time it takes inhibition to wear off is the longest time constant. The inhibition provides a kind of quasi-steady state that is tracked by the voltage of each of the target cells. By the time the inhibition wears off, the cells are essentially at the same voltage, and will fire synchronously, provided the target cells are identical. Thus initial conditions are wiped out by the inhibition.

If the cells are not identical, this mechanism gives phase differences for the firing times of the target cells. If there are other currents involved, as in other rhythms, the effect of the synapses does not necessarily lead to synchronization. A similar mechanism, though harder to understand mathematically, produces a gamma rhythm for a coupled system of equations: the common inhibition here comes from the population (White et al. , 1998; Chow et al. , 1998; Wang \& Buzsaki., 1996). This is called ING or inhibitory network gamma. We will contrast it later 
with another network in which the connections are inhibitory but the cells don't synchronize.

The mechanism for gamma is actually simpler - in principle - when there are excitatory cells involved. Pyramidal Interneuron Network Gamma (PING) is induced in vitro by tetanic stimulation of tissue: the stimulation is higher frequency than gamma; when it is over, the network keeps firing at gamma for a short time, with the excitatory pyramidal cells (E-cells) firing on each cycle, as do the inhibitory fast-spiking interneurons (Whittington et al. , 2000). The PING rhythm is easiest to understand when there is one excitatory and one inhibitory cell (I-cell). The excitation from the pyramid (excitatory cell) causes the I-cells to spike, which inhibits both cells, and the cycle begins again when the inhibition wears off. We think of this as "ping-pong", because the action bounces between the two kinds of cells. The only important currents other than synaptic currents are the standard spiking currents.

Things get more subtle when there is a larger population. One of the subtle aspects is that the synchronization can happen even in extremely sparse and heterogeneous networks, as shown and explained mathematically by Börgers and Kopell (Börgers \& Kopell, 2003; Börgers \& Kopell., 2005). Here it is the I-cells that synchronize their target population, the E-cells, as described above. The E-cells synchronize the I-cells more crudely, but enough to add to the process.

This mechanism also depends on the time scale of decay of inhibition, but not as much (Whittington et al. , 2000). If there are both I-I and E-I connection in the network, changes of parameters can take the network between the ING and PING regimes; in the PING regime, the I-I connections (or even E-E connections) are essentially irrelevant. This has implications for responses to heterogeneity and noise.

\section{A theta-rhythmic cell}

Gamma is the simplest rhythm because it requires no intrinsic currents other than those that produce the spike currents; it is essentially a network rhythm, in which inhibition is critical. By contrast, the theta rhythm is produced by many different kinds of cells in the nervous system. One of these in the area we are focusing on is called the O-LM cell, short for oriens-lacunosum moleculare, for the latin names of the layer of the cell body (oriens) and where it projects to (figure 2). We will refer to it as an O-cell. This single-cell rhythm depends on other intrinsic currents. One of these, which plays a large role in the network behavior, is called the h-current, for heart, where it was found (it is also called "sag current", "weird current", "anomalous rectifier").

The unexpected properties of this current come from its interaction with excitation and inhibition. The interactions have to do with how intrinsic conductances depend on the voltage of the cell. For most conductances, increasing the voltage increases the conductance, i.e., opens the gate more. However, there are some con- 


\section{O-LM interneuron}

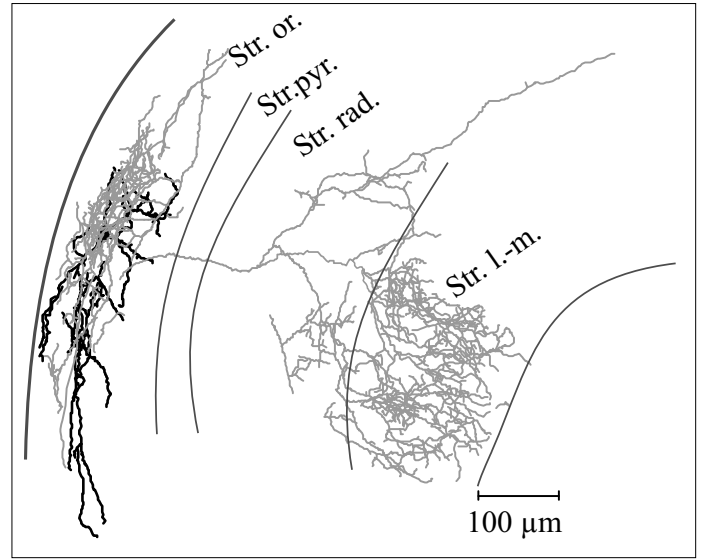

Fig. 2. Reconstruction of an O-LM cell. The dendrites are in str. oriens, and the axons branch into str. lacunosum moleculare, as well as str. oriens.

ductances that act in an anti-intuitive manner: the higher the voltage, the smaller the conductance. This is true of a class of currents known as "hyperpolarization activated currents", which includes the h-current. These currents turn up in many cells in the nervous system, including the O-cell. Indeed, the h-current is the main current that determines the voltage between spikes for those cells (Saraga et al. , 2003; Rotstein et al. , 2005).

When inhibition or excitation is added to a cell that has nonlinear conductances between spikes, it doesn't just change the voltage by adding a new current; it changes the other currents that are sensitive to the voltage. Adding inhibition to the O-cell can initially lower the voltage, but then the h-current turns on and makes the voltage go back up. So inhibition in such a cell can actually make the next spike come faster!

To get a rhythm, cells must fire in a coherent way; one of the mathematical themes of this paper concerns synchronization properties of cells coupled by synapses. As we discuss more below, the h-current has massive effects on network behavior: This current totally reverses the synchronizing properties of excitation and inhibition (Crook et al. , 1998; Ermentrout et al. , 2001; Netoff et al. , 2005; Acker et al., 2003). Biophysical O-cells are inhibitory, and model O cells, when connected with inhibition, do not synchronize for most initial conditions (unlike the more standard inhibitory cells described above); this can be traced to the effects of inhibition on this current. We discuss below how the properties of this current can get built into low-dimensional maps that capture the behavior. 


\section{Structure of Hodgkin-Huxley equations and low-dimensional maps}

The Hodgkin-Huxley equations have structure that sometimes enables one to use low-dimensional approximations, at least near some set of relevant trajectories (Clewley et al. , 2005; Pervouchine et al. , 2006; Rotstein et al. , 2006). For that, we have to recall that the main equation is a conservation of charge, with a sum of different currents. In different voltage ranges, some of these currents are not active, and then the kinetic equations that go with their gating variables are not relevant. Within the active set of currents, some of the gating variables may have time scales that are very long, and hence the relevant variable is essentially locally constant, or very short and hence the variable is slaved to other slower variables. Some kinetics are themselves voltage dependent; gates can be slow to open, but very fast to reset to closed once the voltage gets sufficiently large. That means they reset after a cell spikes, again lowering the number of really independent variables. We use all of these in constructing low-dimensional maps of interacting cells.

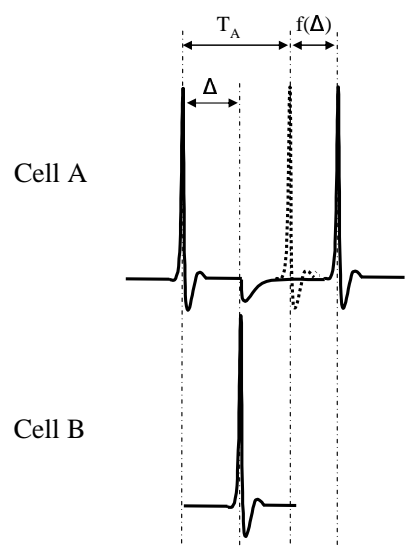

(a)

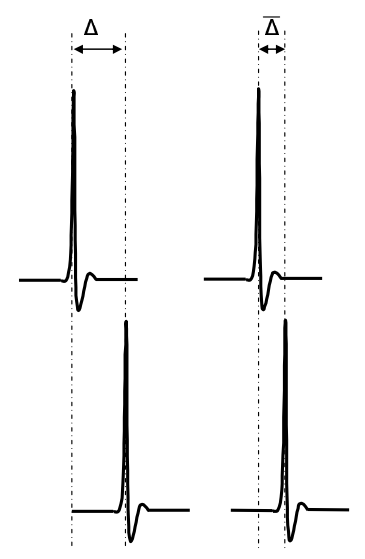

(b)

Fig. 3. Spike time response method. (a) Construction of the spike-time response curve. (b) Construction of the spike-time difference map.

Figure 3 gives the basics of the idea of the lowest possible dimensional maps in question. The first panel shows the idea of the Spike-Time Response Function, 
which is essentially the same as a phase response curve, only explicitly in time rather than phase. Cell B sends a pulse to cell A at some time $\Delta$ after a spike of A, and the STRC measures the change in the time A next spikes compared to when it would have spiked. In the figure, $T_{A}$ is the time it would have spiked, and the difference is called $f(\Delta)$. For this to be at all useful, it has to be checked that no other variable besides $\Delta$ - e.g., the status of any of the gating variables - makes a difference to the time of the next spike. Though we will not discuss that, where we use such formalism, such assumptions generally follow from the reduction ideas sketched above.

Now we consider what happens when two cells interact, and ask if the two cells lock at some phase. We construct a map that takes the time difference between the spikes of the cells on one cycle to the time difference after they have both spiked. There is an extra hypothesis that goes into this: we have to know the order of the spikes to do this construction. In the figure given in panel b, we are assuming that the spikes do not change order. One gets the map by following the effects of each spike on the other cell, starting from a fixed time difference $\Delta$.

The significance of the STRCs and STDMs are that they are the bridges between the biophysics of the cells and their synapses and the behavior of the network. Changing anything biophysical leads to changes in these functions and maps, and allows one to understand how altered biophysics can change the network behavior.

\section{Where does the theta come from?}

We said above that a pair of O-cells, coupled by its own inhibition, does not synchronize. We can understand this from constructing STRCs and STDMs corresponding to the interacting O-cells. The first panel in figure 4 gives an example of STRCs for an O-cell getting input from another O-cell. Both the receiving cell and the input is different from that of a simple I-cell (which is like an integrate-and-fire cell) getting I-input. The receiving cell is different because it has the special currents mentioned (especially the h-current). The input is different because the current from an O-cell lasts several times longer than that from an I-cell. Both of these differences contribute to differences in the STRC compared with that of I cells input to I-cell.

The main thing to notice about the STRC is that an input that arrives soon enough after the receiving cell spikes actually speeds up the next spike. This is the effect of the h-current mentioned before (Dickson et al. , 2000). Later in the cycle, the inhibitory input slows down the next spike. This is shown for different values of the maximal h-current. Note that the larger the h-current, the more of an initial effect there is. One can reason from the kinetics and voltage dependences of the h-current why the STRC looks the way it does, but we do not discuss that here. The second panel (figure 4) is a measurement of STRC using what is known as a "dynamic clamp": A computer acts as one cell, and feeds in the synaptic current at wanted times, while controlling the frequency of the receiving cell (Netoff et al. , 

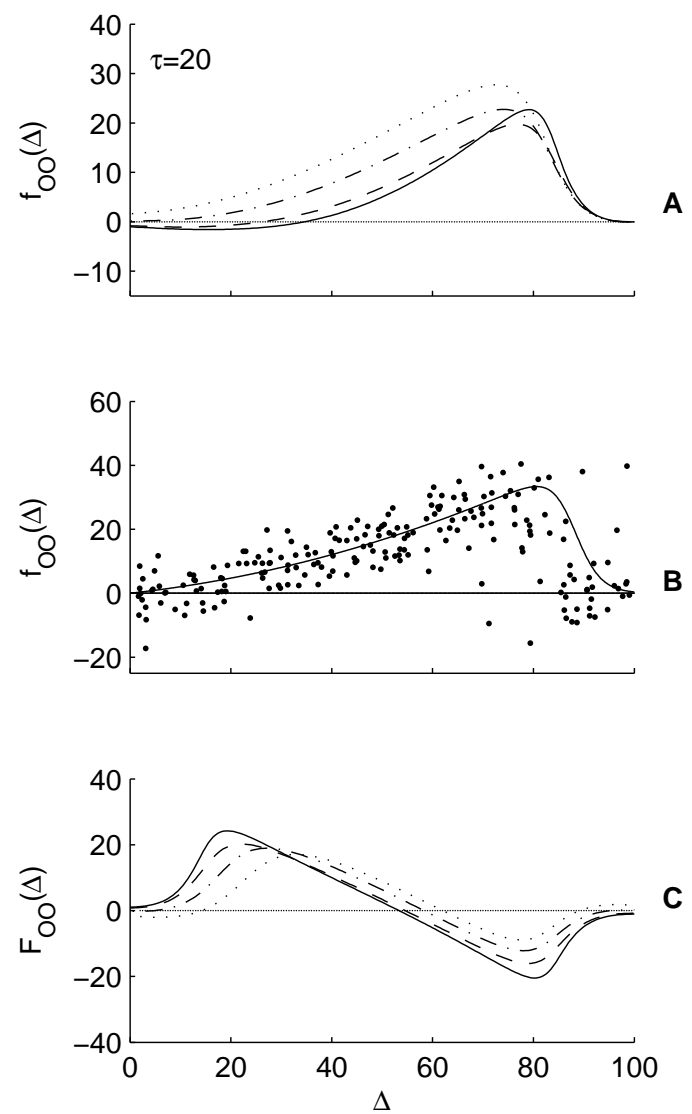

Fig. 4. STRC and STDM for O-O network. (A) STRCs for different levels of the h-current. The solid line corresponds to the highest level of h-current. (B) An experimentally determined STRC. (C) The STDMs constructed from the STRCs in panel A.

2005). Note that it has the same shape as the model STRC, while being considerably noisier.

The next panel (figure 4) gives the associated spike time difference map, also for several values of the h-current. What is shown is not the map itself, but the 
difference $F$ between the map and the identity. Thus, a fixed point for the map is a zero for the function $F$. Recall that a fixed point for a map is stable if the derivative is between -1 and +1 ; this implies that the derivative of $F$ must be between -2 and 0 . The figure shows that there is such stability for a point somewhere in the middle. One cannot tell from this figure, but from symmetry one can reason that this point corresponds to antiphase; the period, which is not the same in the coupled network as in the individual cell, is twice the value of the time at the fixed point of the map.

The punch line of the above is that O-cells, when coupled by their natural coupling, do not synchronize. Hence, it is not possible to get a coherent theta rhythm from a set of O-cells even though each cell is capable of firing at theta; the population rhythm is faster. The same is true for a large number of O-cells. This is in direct contrast to a pair of I-cells, which does synchronize. So, we have a mystery: where does the population theta rhythm come from?

Earlier, we explained how common inhibition can synchronize cells. We also said then that this depends on the cells receiving the inhibition being simple enough. There are I-cells in the CA3 network that produces the theta and one might think that the common inhibition might synchronize the O-cells. But it doesn't necessarily do that. If the I-cell input has the same rough frequency as the O-cells, then it does synchronize them. However, if the input is much faster than the natural frequency of the O-cells, then something else happens, as shown above. Each time there is an inhibitory input, the h-current increases; this partially entrains the O-cells, but cannot make those cells fire much faster than they want to. Instead, the O-cells miss many I-cell cycles, but do fire at a specific phase when they fire. This doesn't synchronize the O-cells because they can skip different cycles (the R. H. panels in figure 5 show the behavior of the h-currents for the two O-cells). Thus, there is not synchronization at the theta frequency.

However, there IS a way to get theta in an $\mathrm{O} / \mathrm{I}$ network that is not intuitively obvious. One needs both the common inhibition to the O-cells and feedback from the O-LM cells to the I-cell. This is un-intuitive, since I-cells can synchronize, but not at theta, and O-LM cells cannot synchronize at any frequency. Figure 6 shows the synchronization. It is critical that the decay time of the O-cells is much longer than that of the I-cells: The long decay time groups the spikes of the I-cell(s) into small bursts. So even when the I-cell is driven enough to fire at high frequencies, one gets the theta rhythm. If there are multiple I-cells so I-cells also get I-inhibition, can get theta and gamma here, nested.

How this works is not intuitive and working with low-dimensional maps helps to explain this. To understand this a little better, we start with just one I-cell and one O-cell. Unlike the O-O circuit described earlier, this is not symmetric. So we don't expect to get synchrony or antiphase, and indeed we don't. The first question is only about whether they lock at all, and at what relative phase.

For this, we can use the techniques described before, using a Spike-Time Response Curves. The first two panels in figure 7 below show those functions, which measure the effects of $\mathrm{I}$ on $\mathrm{O}$ and $\mathrm{O}$ on $\mathrm{I}$. The effects of $\mathrm{I}$ on $\mathrm{O}$ depend on the $\mathrm{h}-$ 

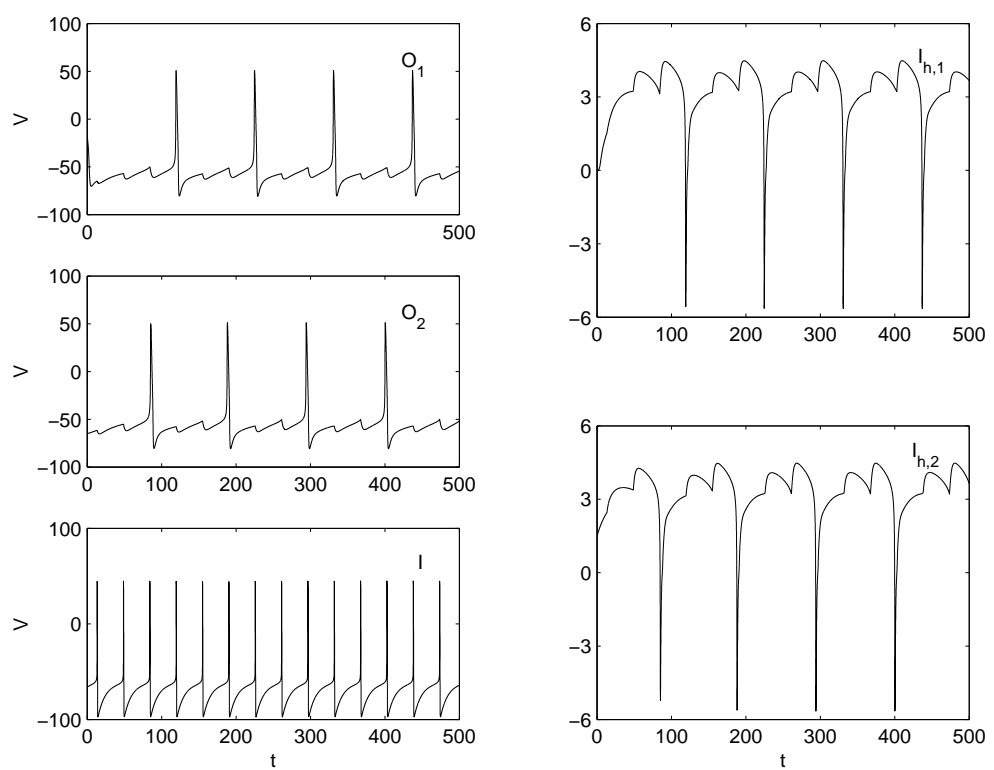

Fig. 5. O-cells need not synchronize with common inhibition. Left panel: The voltages of a pair of O-cells and an I-cell. Right panel: The conductances of the h-current for the two O-cells.
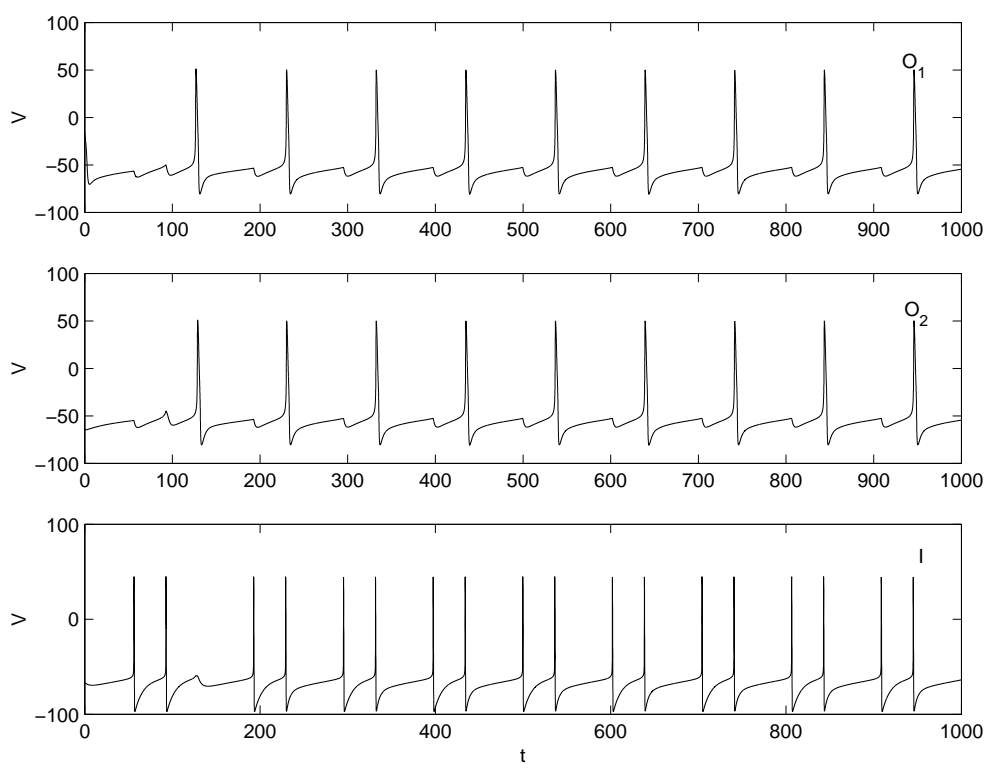

Fig. 6. Adding feedback to the I-cell achieves synchrony of the O-cells. Voltages for the two I-cells and the O-cell. 

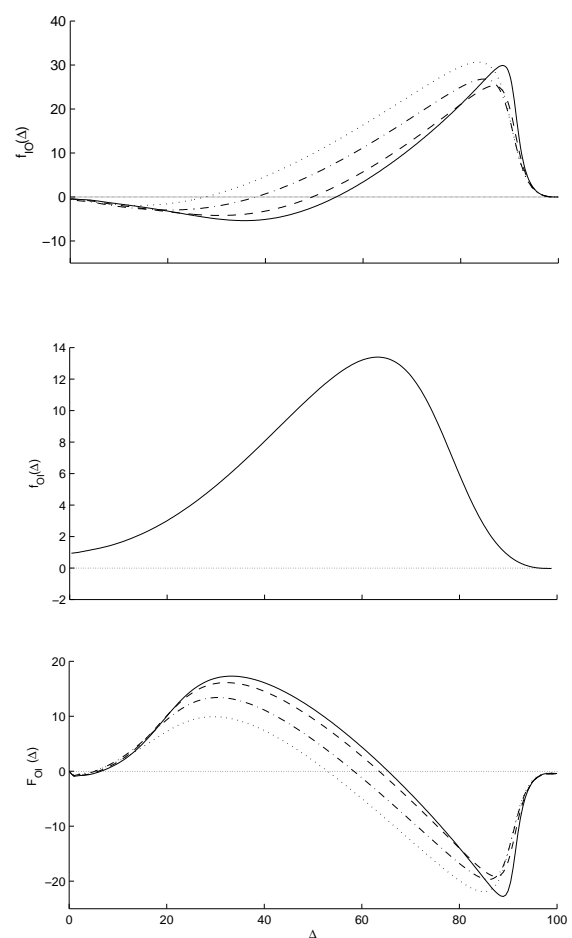

Fig. 7. STRCs and STDM for the O-I network. Top panel: Effect of the inhibition from the I-cell onto the O-cell, at different levels of h-current in the O-cell. Middle panel: Effect of inhibition from the O-cell onto the I-cell. Bottom: STDM giving the difference in time between the firing of the O-cell and the next firing of an I-cell as a function of that difference in the previous cycle.

current in O-cell, so there are several curves. The last panel (figure 7) is the STDM minus the identity, as before. The fixed point of the map, corresponding to the zero of this curve is about 50-70 ms into the cycle, and represents when the I-cell fires after the O-cell in steady state. From the biophysics, we can understand this: The I-cell fires, primes O-cell to fire shortly after the I-cell inhib wears off. The I-cell can fire only after the O-cell inhibition wears off. Both h-current and differences in the decay times lead the interval between I and $\mathrm{O}$ to be considerably shorter than the $\mathrm{O}$ to I interval. In spite of the fact that there are many variables in the relevant equations, a 1-D map is accurate because the variables are slaved to the spike times, even the h-current, which resets whenever the O-cell fires.

One might ask what kind of understanding one gets by thinking about maps, beyond just simulating and seeing what happens. The answer is that it helps us understand what matters to the synchronization of the O-cells, in particular that the long kinetics of the O-cell-derived inhibition is critical. 
Once we have more than one O-cell, we cannot use 1-D maps - there is more than one real degree of freedom, not slaved to one spike time difference. With two O-cells, the system can be described by a 2-D map, with variables $\Delta$, the difference between the O-cell spikes, and $\sigma$, the difference from the second O-cell spike to the I-cell spike. This can be analyzed as a 2-D map, but the essential insight comes from looking at slices of that map gotten by fixing $\sigma$. Figure 8 shows several such slices. Unlike our other graphs, where we are interested in only fixed points, we now draw this as a map, i.e., without taking away the identity, so we can use standard cobwebbing (a graphical method for understanding the dynamics of iterated maps) to see the behavior.

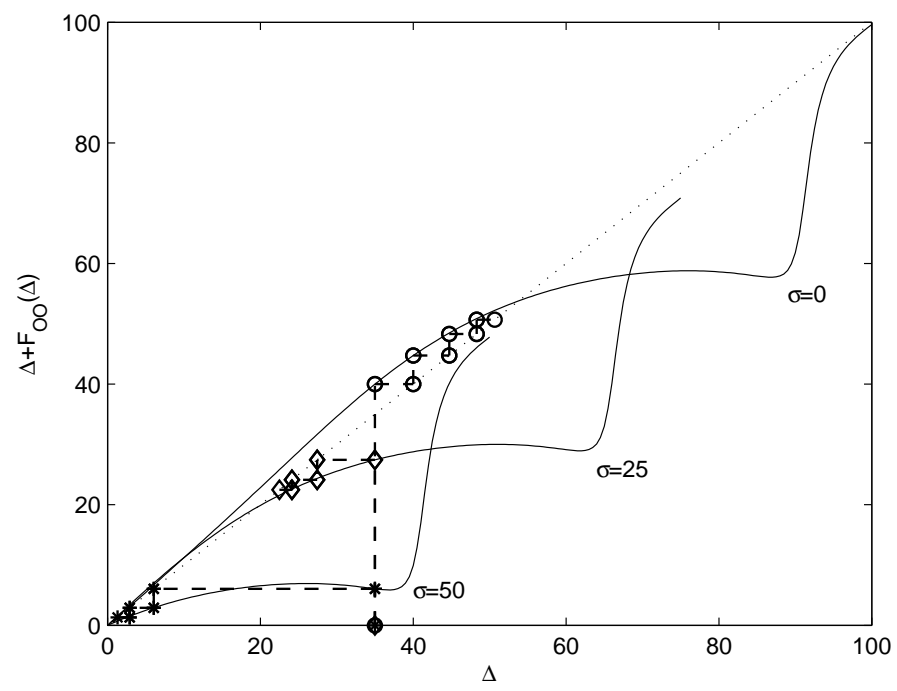

Fig. 8. Slices of 2-D map for fixed $\sigma$

The lowest graph is for $\sigma=50$, and we see that the $\Delta$ goes to zero and stays there by the first cycle. By contrast, the ones with a much smaller $\sigma$ do not go to zero: for $\sigma=25$, the $\Delta$ stays near a much higher value for many cycles, and for $\sigma=0$, the O-cells actually move further apart. Though the slices do not capture the full behavior of the 2-D map, they do accurately measure steady-state behavior, and this shows that, unless the $\sigma$ is large enough, one does not get synchrony of the O-cells as a steady state. The value of $\sigma$ is directly tied to the decay time of the O-cell inhibition, and decreases when the latter decreases. Thus, it is necessary to have the O-cell decay time long enough to make the whole network produce theta when there is more than one O-cell. 


\section{All together now: a larger network}

We now return to the original question: why is it that when CA3 is cut into slices of different orientation, one sees different rhythms? The clue to this comes from the anatomical work that was done by Gloveli and collaborators. The key observation is the way that basket cells (I-cells) and O-cells are arranged. The basket cells tend to arborize more in the plane of the standard transverse slice, the one that gives the gamma rhythm; the O-cells, by contrast, tend to arborize in clumps along the longitudinal axis. In the transverse plane, there is only one clump, but in the longitudinal plane, there are 2-3 separate clumps.

Our question: could this be enough to account for the differences in rhythms? This is an obvious modeling question. We considered building a $3-\mathrm{D}$ simulation to test this, but we believe that dynamical structures are most easily understood in "minimal" models. So what IS minimal here? Our guess, which our simulations support, is that what matters is the relative strengths of the $\mathrm{O}$ - and I-cell projections on the excitatory neurons; we could test this in a model that did not have much, if any, spatial structure. The model network is shown in figure 9. It has a pair of O-cells, a pair of I-cells and a single E-cell that has a soma and a dendrite. We felt that the 2-compartments were important, since the I-cells project to the soma and the O-cells to the ends of the dendrite. Within this model, it was possible to alter the relative strengths of the synapses from the O- and I-cells.

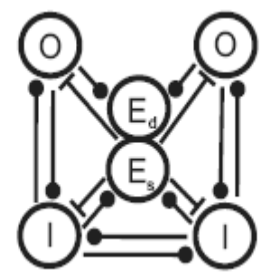

Fig. 9. Schematic of model for nesting of gamma and theta rhythms.

The simulations correspond to the transverse, longitudinal and coronal slices in terms of strengths of connections. The first panel (A) in figure 10 shows the E-cell soma producing a gamma rhythm interacting with the I-cells (which are synchronous). This is a standard PING. The O-cells, which are not synchronous, fire at a lower frequency, producing a small theta envelope to the amplitude of the synaptic currents, as seen in the experiment. To get this rhythm, the parameters are set so that the I to E connections are relatively strong, as are the I-O connections. The O-cells are firing at roughly theta frequency because the h-current builds up in the cells over several gamma cycles, due to the I-cell inhibition, as understood from the interaction between the I and $\mathrm{O}$ cells. As discussed before, the common 
inhibition need not synchronize the O-cells. This is also seen in the experimental data.

To get the theta oscillations shown next, we model the input from the extra clumps of O-cells as increasing the input from the O-cells to the I- and E-cells, and decreasing the input from the I-cells (Panel B). This increasing inhibition from the O-cells, with its much longer decay time, changes the firing of the E-cells to much slower, which then contributes to slowing down the I-cells. The critical switch in behavior is that the I-cells now fire at theta, not gamma. The interaction of the Iand O-cells produces the almost synchrony of the O-cells; if the O-cells had been identical, they would be synchronous, but to match the data, which is more ragged, the cells are not identical in their drives or inputs from the I-cell. The order of the firing - O-cells, I-cell and E-cells, match that in the data. Panel C is the simulation of the coronal slice, in which there is both gamma and theta in the excitatory cells, which are the output cells of the network. There is now a nesting of the gamma and theta, as seen in the experimental data, with the longer period that of theta, and the frequencies in each cluster of spikes gamma. That is achieved just by using intermediate parameters. Recall that one obtains multiple I-spikes for each O-spike provided that the I-cells are sufficiently excited. This is compatible with getting only one spike, as in panel B, when the O-I inhibition is strong, but when that is decreased, the nested rhythm can pop up.

\section{Multiple rhythms, switches and bursting}

The last set of simulations (panel $\mathrm{C}$ ), with the nesting gamma and theta, is very reminiscent of bursting systems. In its simplest form, bursting comes from a single neuron in which there is a structure of a reduced $\mathrm{H}-\mathrm{H}$ equation in 2 dimensions, plus a third variable which is much slower. As the third variable, thought of as a parameter, changes, the 2-D system can change between having a critical point and a limit cycle (Izhikevich, 2006).

The system we are considering has many more variables than 3 , and minimally 3 different cells (one O, one I and one E). However, using reduction of dimension ideas, the effective dimension at any given time is actually much lower. Furthermore the behavior is very similar to classic bursting: the E/I network produces the gamma oscillation when the inhibition from the O-cell, which is slower to decay, is low enough to allow it. Thus, we hypothesize that the switching behavior associated with the three different kinds of behavior shown in figure 10 can be understood in a way similar to that of bursting, using reduction of dimension ideas. This is currently in progress.

\section{Discussion}

The gamma and theta rhythms are present together in various parts of the nervous system under various behavioral situations. We focus on a slice preparation that can produce either gamma or theta or a combination of them both. The same network 

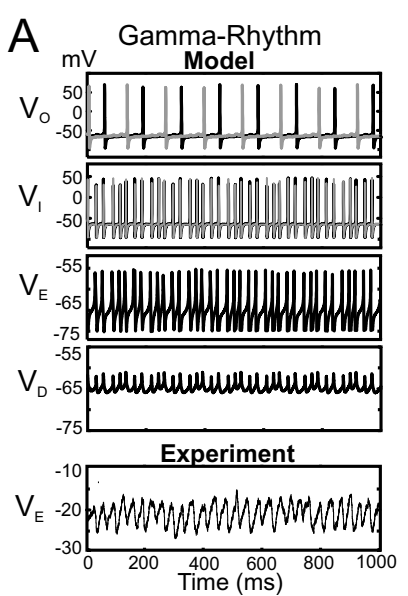
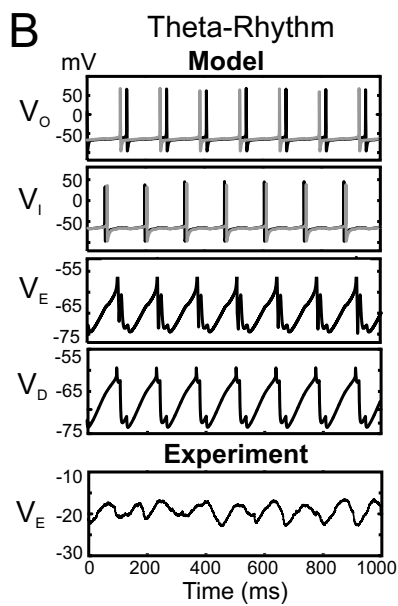

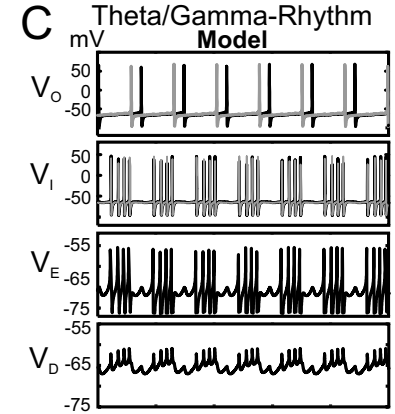

Experiment

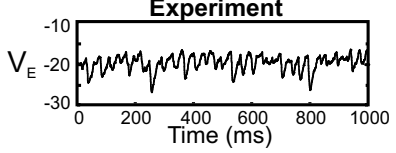

Fig. 10. Simulations of model. (A) the I- and E-cells produce a gamma rhythm. (B) All cells produce a theta rhythm. (C) Gamma rhythm nested inside a theta rhythm.

is involved in all three situations; changes of parameters change which subnetworks control the behavior of the full network. For gamma, the centrally important subnetwork is the E/I one; for theta it is the $\mathrm{O} / \mathrm{I}$ interaction. By changing parameters one can get different subnetworks to dominate the rhythm. The interaction of the rhythms is reminiscent of bursting, in which the state of the fast variables depends on the state of some slower one.

The key functional issue is how the theta oscillation might be useful for coordinating temporal sequences of gamma-induced cell assemblies. It is known that gamma rhythms are local, while theta coherence is more global (Gloveli et al. , 2005). It is also known (Jalics, Kispersky and Kopell, unpublished observation) that theta interacts with gamma, and theta-frequency inputs can change the phase of gamma rhythms. The understanding of the biophysical bases of gamma and theta rhythms provides clues for how the networks react to input that is spatially as well as temporally structured.

\section{References}

1. Acker, C., Kopell, N., \& White, J. 2003. J. Comp.Neurosci, 15, 71.

2. Börgers, C, \& Kopell, N. 2003. neurocomp, 15(3), 509-538.

3. Börgers, C., \& Kopell., N. 2005. Neural Computation, 3, 557.

4. Chow, C., White, J., Ritt, J., , \& Kopell, N. 1998. J. Comput. Neurosci., 5, 407.

5. Chrobak, J., Lorincz, A., \& Buzsaki., G. 2000. Hippocampus, 10, 457.

6. Clewley, R., Rotstein, H., \& Kopell., N. 2005. Multiscale Modeling and Simulation, 4, 732 .

7. Crook, S., Ermentrout, G. B., \& Bower, J.M. 1998. Neural Computation, 10, 837. 
8. Dayan, P., \& Abbott, F. 2005. Theoretical Neuroscience: Computational and Mathematical Modeling of Neural Systems. MIT press.

9. Dickson, C., Magistretti, J, Shalinsky, M.H., Fransén, E., Hasselmo, M.E., \& Alonso, A. 2000. Journal of Neurophysiology, 83, 2562.

10. Ermentrout, B., Pascal, M., \& Gutkin., B. 2001. Neural Computation, 13, 1285. 11. Gloveli, T., Dugladze, T., Rotstein, H., Traub, R., Heinemann, U., Monyer, H., Whittington, M., \& Kopell, N. 2005. Proc. Nat. Acad. Sci., 102, 13295.

12. Izhikevich, E. 2006. Dynamical Systems in Neuroscience: The Geometry of Excitability and Bursting. MIT press.

13. Netoff, T., Banks, M., Dorval, A., Acker, C., Hass, J., J., N. Kopell, \& White, J. 2005. Journal of Neurophysiology, 93, 1197.

14. Pervouchine, D., Netoff, T., Rotstein, H., Cunningham, M., Whittington, M., White, J., \& Kopell, N. 2006. Neural Computation.

15. Rotstein, H., Gillies, M., Acker, C., White, J., E.Buhl, Whittington, M., \& Kopell, N. 2005. J. Neurophysiol, 94, 1509.

16. Rotstein, H., Oppermann, T., White, J., \& Kopell., N. 2006. J. of Comput. Neurosci.

17. Saraga, F, Wu, C P, Zhang, L, \& Skinner, F K. 2003. J. Physiol, 552, 502.

18. Wang, X. J., \& Buzsaki., G. 1996. J. Neurosci., 16, 6402.

19. White, J., Chow, C., Ritt, J., Soto-Trevino, C., \& Kopell, N. 1998. J. Comput. Neurosci., 5, 5-16.

20. Whittington, M.A., Traub, R.D., Kopell, N., Ermentrout, G.B., \& Buhl, E.H. 2000. Int. J. of Psychophysiology, 38, 315-336. 